\title{
A Scalable Distributed Video Coder using Compressed Sensing
}

\author{
Md Mashud Hyder and Kaushik Mahata \\ School of Electrical Engineering and Computer Science, University of Newcastle, \\ Callaghan, NSW 2308, Australia. \\ md.hyder@studentmail.newcastle.edu.au,kaushik.mahata@newcastle.edu.au
}

\begin{abstract}
Distributed Video Coding (DVC) is a new paradigm for video compression, which is particulary attractive due to its flexibility to introduce very simple encoder structure. However, most DVC systems are unable to adapt to the bandwidth variations. In this paper we propose a scalable DVC scheme, which can adjust the bit-rate linearly depending on the available bandwidth, and thereby achieves optimal quality of service (QoS). Our algorithm combines compressed sensing with multiple measurement vectors problem to achieve significantly low encoding complexity. The correlation structure in the successive video frames is exploited by joint sparsity, which in turn helps reduce decoding complexity. The simulation results depict a significantly improved performance over other low complexity video codec schemes.
\end{abstract}

Keywords- Distributed video coding, Wyner-Ziv coding, Sparse representation, multiple measurement vectors, joint-sparse. compressive sampling

\section{INTRODUCTION}

Traditional digital signal compression paradigm is typically a two step process. In the first step the signal is passed through a predictor to generate the innovation sequence made up of the one-step prediction errors. Subsequently, the innovations are quantized using some suitable quantizer. In the conventional video coding architectures such as MPEG-x and H.26x, the prediction step is computationally intensive with the complexity dominated mainly by motion estimation and detection operations. On the other hand, video decoding is of significantly lower complexity. This asymmetry is well-suited for broadcasting or for streaming video-on-demand systems where video is compressed once and decoded many times. However, with the advances in contemporary technologies, many emerging applications demand low complexity encoder, in particular for mobile reception devices. Examples of such devices include low cost single-use video cameras, hand held multimedia devices and security surveillance systems. The increasing popularity of such devices has motivated distributed video coding (DVC). DVC relies on two major information theory results due to Slepian-Wolf [1] and Wyner-Ziv [2]. These suggest that two statically dependent discrete random signals $X$ and $Y$ can be independently encoded, and still be jointly decoded with an

Research is supported by the Australian research council. arbitrary small error probability provided that

$$
R_{X} \geq H(X \mid Y), R_{Y} \geq H(Y \mid X), R_{X}+R_{Y} \geq H(X, Y)
$$

where $R_{X}$ and $R_{Y}$ represent the rates for encoding $X$ and $Y$; $H(X, Y)$ is the joint entropy, and $H(X \mid Y)$ and $H(Y \mid X)$ are the conditional entropies of $X$ and $Y$. Using this idea DV encodes a video frame independently of the neighboring frames, while the decoder exploits the underlying correlation among the frames. As DVC exploits the source statistics in the decoder, the majority of the complexity is shifted to decoder.

Scalability is a highly desired merit of a video coder [3]. It plays a crucial role in forth generation mobile networks. As the channel condition is ever changing, the physical layer adapts by so called 'link adaptation'. The idea is to adjust the transmission rate according to the signal to noise ratio (SNR) and level of contention. This in turn requires adjusting the bit-rate of the underlying signal source coders. The ISO/IEC MPEG-4 standard provides flexible scalability tools, including spatial and temporal and SNR scalability. A major problem, however, is that the standard video coders are often unable to increase or decrease the bit-rates in finer steps. For example, suppose we need to decrease the bitrate only by $5 \%$. However, then the underlying video coder has to decrease the bit-rate at least by $20 \%$. As a result the network bandwidth remain unutilized, while the quality of reconstructed video becomes poor leading to unsatisfactory QoS.

In this paper we propose a scalable DVC based on compressed sensing (CS) [4], [5]. The proposed coding scheme can adjust the bit-rate in very fine steps. In addition, this scheme offers extremely low encoding complexity. The decoder exploits the statistical dependency among successive frames by formulating a multiple measurement vector (MMV) problem [6], [7]. The proposed codec does not strictly follow the DVC architecture. Nevertheless, it builds on the philosophy of transferring the complexity from the encoder to the decoder.

\section{PRoposed DVC SCHEME}

\section{A. Background}

In the following we describe the basic structure of a DVC system [8], [9]. A video is a temporal sequence of images (also called frames). The key frames constitute a subset of the frames, regularly spaced in time. A key-frame is encoded and decoded using a conventional image codec (e.g. a discrete cosine transform (DCT) beased codec). The number of frames between two consecutive key-frames depends on the DVC coder, and is called the Group of Pictures (GOP). These frames between key-frames are called 
the Wyner-Ziv frames. The Wyner-Ziv frames are encoded using very low complexity encoders, where temporal correlation between consecutive fames are ignored. Typically this is done by passing the Wyner-Ziv frames via a Turbo encoder.

At the decoder the key-frames are decoded using the standard image decoder. However, the process of reconstructing the the Wyner-Ziv frames does take the temporal interframe correlation into account. This is done using some side information predicted from the key-frames. Coding of Wyner-Ziv frames can be done in various ways. These include motion prediction based approaches, relatively simple pixel-domain approaches, transform coding methods (see [9] and references therein), and compressive sensing based techniques [10], [11].

\section{B. Proposed DVC Encoder}

In the following the Key-frame and Wyner-Ziv frames are indicated by using the subscripts $I$ and $W$, respectively. Each key frame is encoded by an H.264/AVC encoder independent of any other frame. A Wyner-Ziv frame $x_{W}$ is encoded into a measurement vector $y_{W}$ using the techniques of compressive sensing as explained below.

Consider the vectorized version $x \in \mathbb{R}^{n}$ of an image. If the original image $\mathcal{I}$ was a $n_{1} \times n_{2}$ intensity map we obtain $x=\operatorname{vec}\{\mathcal{I}\}$, and $n=n_{1} n_{2}$. Now it is well known that there exists a basis $\left\{\psi_{t}\right\}_{t=1}^{n}$, which provides a sparse representation of $x$, i.e. $x$ admits a representation $x=\psi_{1} c_{1}+\cdots \psi_{n} c_{n}$ where only a few of the expansion coefficients $c_{k}$ are non-zero. The basis could be a DCT basis or a wavelet basis. The existence of the sparse representation allows compressive sensing. The measurement vector

$$
y=\Phi x,
$$

where $\Phi \in \mathbb{R}^{M \times n}$ is typically a random matrix, $y$ contains all information about $x$. Here $M<n$. Hence the dimension of $y$ is significantly smaller than that of $x$. The proposed encoder encodes the Wyner-Ziv fames into such compressive linear random measurements. The random matrix $\Phi$ is formed using noiselets, see [5] for details. The encoding process of a single GOP is summarized below

1) Encode keyframe using H264/AVC encoder. Output $z_{I}$. Construct measurement matrix $\Phi \in \mathbb{R}^{M \times n}$.

2) Form measurements $y_{W}^{(i)}=\Phi\left(x_{W}^{(i)}-x_{I}\right)$, where $x_{W}^{(i)}$ is the $i$-th Wyner-Ziv frame in the GOP.

3) Quantize $y_{W}^{(i)}, i=1, \ldots, L$, using an appropriate quantization scheme. Where $L$ is the number of Wyner-Ziv frames in a GOP.

The key-frame $x_{I}$ subtracted from each $x_{W}$ in order to exploit the temporal correlation structure. This helps to reduce the bitrate. The quantizer is designed using Lloyd's algorithm [12]. The bit rate for each component of $y$ is 2 bits.

\section{The Decoder}

Decoding of the key frame $x_{I}$ from $z_{I}$ is straightforward, the standard H264/AVC decoder is employed. It is assumed that the decoder have information about the measurement matrix used in encoder. To decode Wyner-Ziv frame, decoder requests $m \leq M$ components of $y_{W}$ from encoder. The size of $m$ depends on available bandwidth. Based on the size of $m$ the decoder can construct $\Phi \in \mathbb{R}^{m \times n}$ using first $m$ rows of the measurement matrix used in encoder. Note that the decoded version $\hat{x}_{I}$ is not identical to $x_{I}$. Then the encoding procedure suggests that we can write

$$
\widehat{y}_{W}^{(i)}+\Phi \hat{x}_{I}=\Phi x_{W}^{(i)}+e_{i},
$$

where $\widehat{y}_{W} \in \mathbb{R}^{m}$ and $e_{i}$ is a small residual term which absorbs the errors due to quantization and noise effects. Note that the left hand side of (2) is known. However, (2) alone is not sufficient for estimating $x_{W}^{(i)}$. This is because $\Phi$ has more columns than rows. To deal with this issue we resort to compressive sensing literature. In particular we set up a so-called multiple measurement vector framework. Repeating (2) for $i=1,2, \ldots, L$ and arranging in matrix form we have

$$
Y=\Phi X+E,
$$

where $E$ is the contribution from the residuals and

$X=\left[\begin{array}{lll}x_{W}^{(1)} & \cdots & x_{W}^{(L)}\end{array}\right], \quad Y=\left[\begin{array}{lll}\widehat{y}_{W}^{(1)}+\Phi \hat{x}_{I} & \cdots & \widehat{y}_{W}^{(L)}+\Phi \hat{x}_{I}\end{array}\right]$.

In order to arrive at the desired solution $X$ we exploit the sparsity structure of $X$. This is done via total variation (TV) minimization [13]. Let $D_{h}$ and $D_{v}$ be the Eular's first order difference operators in the horizontal and vertical directions, respectively. Then the image $D_{h} x$ shows the horizontal edegs in $x$, while the image $D_{v} x$ shows the vertical edegs. Then both $D_{h} x$ and $D_{v} x$ are sparse, because an image contains only a few edges typically. Furthermore, if $\left[D_{h} x\right]_{k}$, the $k$-th component of $D_{h} x$, has a significant magnitude, then there is an edge in the pixel corresponding to the index $k$. Then one would expect a significant magnitude of the vertical derivative at that location making $\left|\left[D_{h} x\right]_{k}\right|$ large as well. Hence the matrix $\left[\begin{array}{cc}D_{h} x & D_{v} x\end{array}\right]$ is jointly row sparse, meaning that only a few rows of this matrix have significantly large 2-norm. While this also holds for the Wyner-Ziv frames $\left\{x_{W}^{(i)}\right\}_{i=1}^{L}$, there are more stuructures due to strong temporal correlation. In fact, if $L$ is small (which is the case) then the matrix

$$
Z:=\left[\begin{array}{ll}
D_{h} X & D_{v} X
\end{array}\right]
$$

turns out to be jointly row sparse. To understand this consider a $k$ such that $\left|\left[D_{v} x_{W}^{(i)}\right]_{k}\right|$ and $\left|\left[D_{k} x_{W}^{(i)}\right]_{k}\right|$ are large, then there must be an edge in the pixel corresponding to the index $k$. However, the frames $x_{W}^{(i-1)}, x_{W}^{(i+1)}$ and other neighboring frames are very close to $x_{W}^{(i)}$. Hence one would expect the presence of the same edge in the pixel corresponding to the index $k$ in all the neighboring frames. Thus $\left|\left[D_{v} x_{W}^{(i+1)}\right]_{k}\right|$ and $\left|\left[D_{k} x_{W}^{(i+1)}\right]_{k}\right|$ are large, and so are $\left|\left[D_{v} x_{W}^{(i-1)}\right]_{k}\right|,\left|\left[D_{k} x_{W}^{(i-1)}\right]_{k}\right|$, etc. Hence $Z$ in (4) is jointly row sparse.

The ideal approach to recover $X$ by imposing the joint sparse structure on $Z$ is to minimize

$$
\min _{X}\|Z\|_{p, q}+\frac{\lambda}{2}\|Y-\Phi X\|_{F}^{2}
$$

for $p=2$ and $q=0$, where $\|A\|_{F}^{2}=\|\operatorname{vec}(A)\|_{2}^{2}$ is the Frobenious norm of $A$; and the mixed norm $\|Z\|_{p, q}$ is defined as

$$
\|Z\|_{p, q}=\left(\sum_{j=1}^{n}\left(\sum_{i=1}^{2 L} Z[j, i]^{p}\right)^{q / p}\right)^{1 / q},
$$

with $Z[i, j]$ being the entry of $Z$ at $i$-th row and $j$-th column. The parameter $\lambda$ controls the tradeoff between the sparsity of the signal and the residual energy [14]. 
TABLE I: JLZA-TV Algorithm

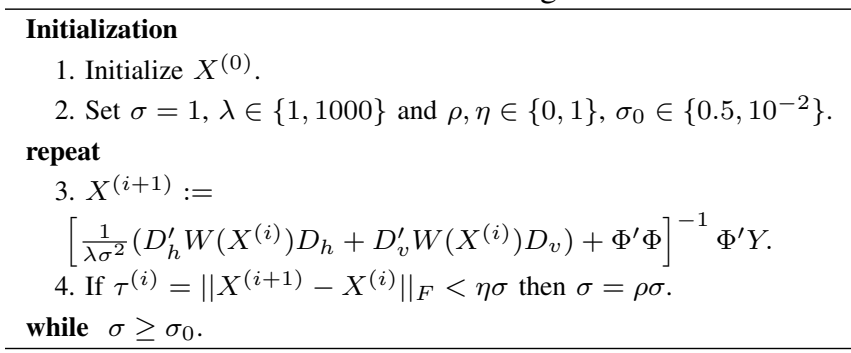

Since the choice $p=2, q=0$ leads to a NP-hard problem, several relaxations have been proposed [6], [15]. Here we follow JLZA [7] which approximates $\|Z\|_{2,0}$ via a sequence of Gaussian functions [16]. Denote the $j$-th row of $Z$ by $Z[j,:]$, and define

$$
F_{\sigma}(Z):=\sum_{j=1}^{2 L} f_{\sigma}\left(\|Z[j,:]\|_{2}\right), \quad f_{\sigma}(v)=\mathrm{e}^{-v^{2} /\left(2 \sigma^{2}\right)} .
$$

It can verified that [7] $F_{\sigma}(Z)$ behaves like $n-\|Z\|_{2,0}$ when $\sigma \rightarrow 0$. This motivates the following way to estimate $X$. Let

$$
\begin{aligned}
& X_{*}(\sigma)=\arg \min _{X} \quad L_{\sigma}(X), \\
& L_{\sigma}(X):=-F_{\sigma}(Z)+\frac{\lambda}{2}\|Y-\Phi X\|_{F}^{2} .
\end{aligned}
$$

Just like $\|X\|_{2,0}$, its approximation $n-F_{\sigma}(X)$ has many local minima for small values of $\sigma$. Nevertheless, as $\sigma$ increases, $F_{\sigma}$ becomes smoother. In fact $\lim _{\sigma \rightarrow \infty} F_{\sigma}(\alpha)=1$. Consequently, Using this in (6) we have

$$
\lim _{\sigma \rightarrow \infty} X_{*}(\sigma)=\Phi^{\prime}\left(\Phi \Phi^{\prime}\right)^{-1} Y
$$

Hence, we take a large $\sigma$ initially and solve (6). Subsequently, $\sigma$ is reduced by some small factor and (6) is solved again. The procedure is repeated until a small value of $\sigma$ is reached. It can be shown that

$$
\begin{gathered}
\frac{\partial L_{\sigma}(X)}{\partial X}=\frac{1}{\sigma^{2}}\left\{D_{h}^{\prime} W(X) D_{h}+D_{v}^{\prime} W(X) D_{v}\right\} X-\lambda \Phi^{\prime}(Y-\Phi X) \\
W(X)=\operatorname{diag}\left\{f_{\sigma}\left(\|Z[1,:]\|_{2}\right), \ldots, f_{\sigma}(\|Z[n,:]\|)\right\} .
\end{gathered}
$$

Hence it follows that

$$
\begin{gathered}
X_{*}(\sigma)=\zeta\left\{X_{*}(\sigma)\right\}, \\
\zeta(X):=\left[\frac{1}{\lambda \sigma^{2}}\left(D_{h}^{\prime} W(X) D_{h}+D_{v}^{\prime} W(X) D_{v}\right)+\Phi^{\prime} \Phi\right]^{-1} \Phi^{\prime} Y .
\end{gathered}
$$

We solve (7) using a fixed point iteration. The outline of the algorithm to solve $X$ is given in Table I. In the algorithm, $X^{(i)}$ denotes the value of $X$ updated at $i^{\text {th }}$ iteration. The initialization process of $X^{(0)}$ is described below. We set $\sigma=4 \max _{i}\left\|Z^{(0)}[i,:]\right\|_{1}$ for the first iteration [16]. The value of $\lambda$ depends on noise level. Experimental results suggests that $\lambda=1000$ is good choice for the available video sequences. For minimizing $L_{\sigma}$ we use (8) as a fixed-point iteration. The inner-iteration for a given $\sigma$ terminates when $\tau^{(i)}<\eta \sigma$, (see step 4). A wide range of numerical experiments suggests that the good setting of $\eta$ is 0.5 . The stopping criterion of JLZA-TV is based on a small $\sigma$ denoted by $\sigma_{0}$. Upon convergence of each inner iteration we lower $\sigma$ by a factor $\rho$ (step 6 ). When $\sigma$ becomes equal to $\sigma_{0}$, we do not update $X^{(i)}$ further and stop iterations. The final value of $\sigma_{0}$ is set to 0.1 and $\rho=0.3$.

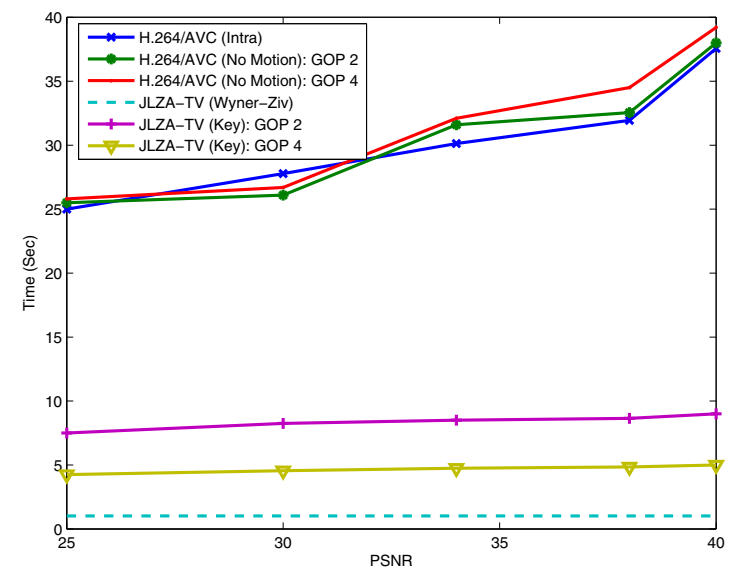

Fig. 2: Encoding time comparison for Foreman test sequence.. $\mathrm{GOP}=2$.

1) Initialization of $X^{(0)}$ : The convergence time of (6) using the algorithm in Table I depends on initializer of $x^{(0)}$. Since we already have the key frame $\hat{x}_{I}$ hence it is natural to choose $X^{(0)}$ such that

$$
X^{(0)}[:, i]=\operatorname{argmin}_{x}\left\|x-\hat{x}_{I}\right\|_{2}^{2} \quad \text { subject to } \widehat{y}_{W I}=\Phi x
$$

where $\widehat{y}_{W I}=\hat{y}_{W}+\Phi \hat{x}_{I}$. It can be shown that

$$
X^{(0)}[:, i]=\hat{x}_{I}+\Phi^{\prime}\left(\Phi \Phi^{\prime}\right)^{-1}\left(\widehat{y}_{W I}-\Phi \hat{x}_{I}\right)
$$

\section{Scalability}

Scalability of the algorithm results from the fact that the number of rows $m$ of $\Phi$ can be flexible. A large $m$ gives better accuracy of reconstruction. However, it is found that if $m_{l} \leq m \leq m_{u}$ reconstruction is still possible with some loss. Moreover the reconstruction quality changes almost linearly with $m$. Hence depending on the available bandwidth we adjust $m$. Accordingly the reconstruction quality is adjusted.

\section{Simulation Results}

We present the results obtained from the Foreman (with Siemens logo) test video sequence (QCIF format). We use first 150 frames and frame rate is 15 frames/second. The PSNR and bit rate results are averaged over the sequence. The performance of the proposed codec is compared with H.263+ intra, H.264-Intra frame coding (profile: main, v10.1) and H.264-No motion frame coding.

Figure 1 depict the PSNR performance comparison. Note that the PSNR of JLZA-TV improves rapidly with bit rate increase. Note that H.264/AVC (Intra) performs well for low bit rate. However, it can support only a few predefined bit rates . For example, in Fig. 1a the PSNR is 34.75 at $248.82 \mathrm{Kbps}$ and 38 at $397.28 \mathrm{Kbps}$. As it supports no intermediate bit-rate, we need to switch to $248.82 \mathrm{Kbps}$ even if we want to lower the bit-rate slightly below below $397 \mathrm{Kbps}$, resulting poor utilization. On the other hand JLZA-TV offers a step-length of $20 \mathrm{Kbps}$, and in effect allows better utilization, hence improved PSNR and QoS. 


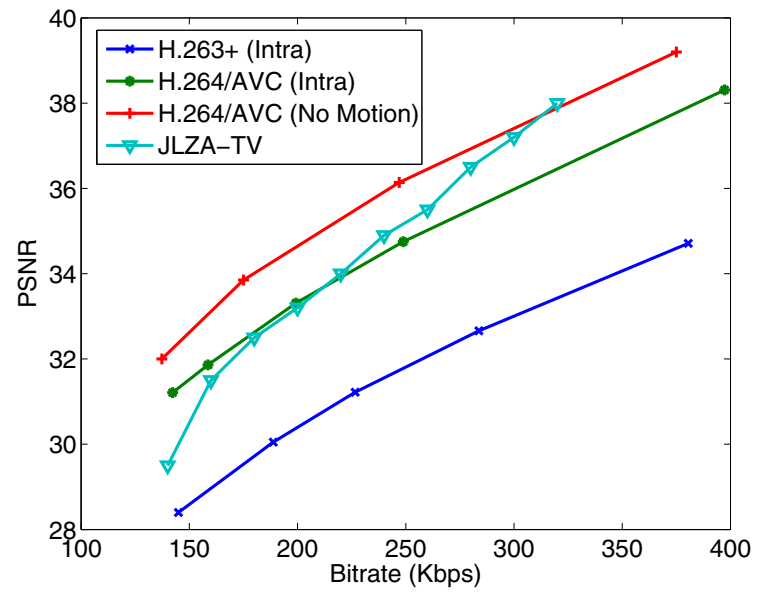

(a) Performance comparison for Foreman test sequence. GOP $=2$.

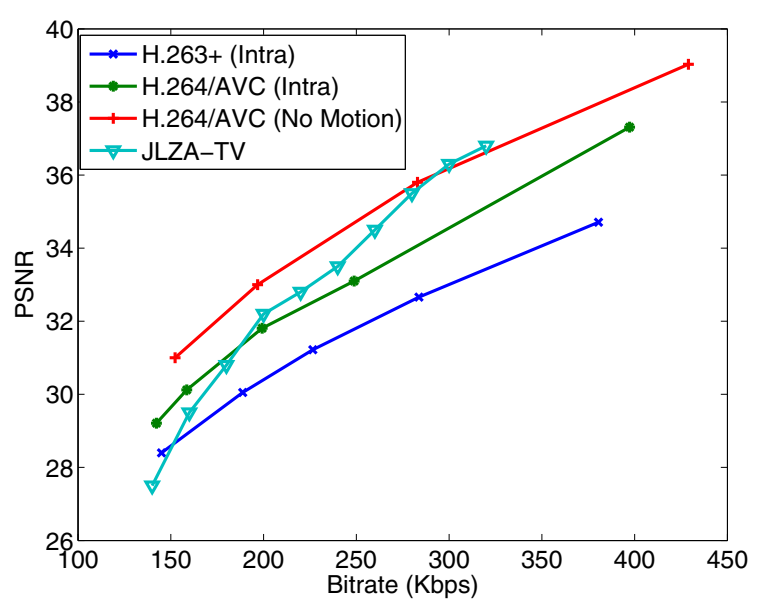

(b) Performance comparison for Foreman test sequence. GOP $=4$.

Fig. 1: Rate-Distortion results for Foreman sequence.

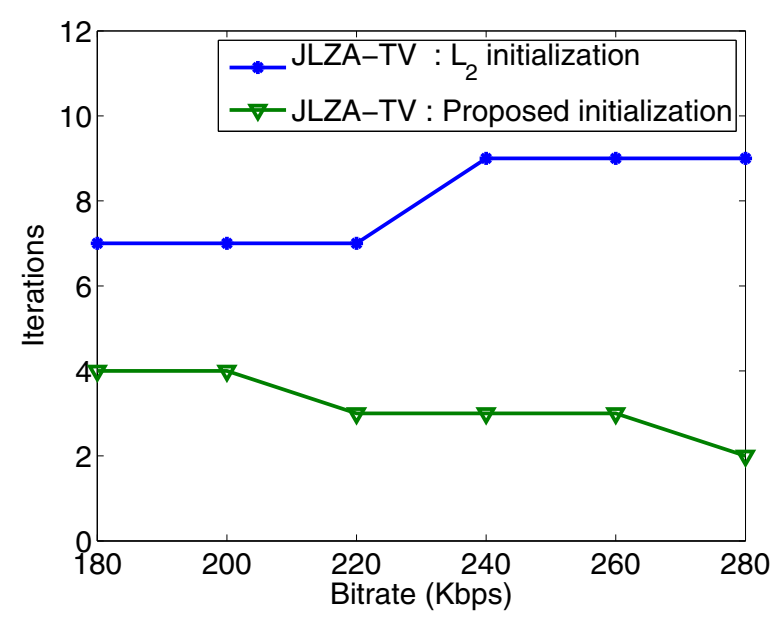

Fig. 3: Effect of proposed initialization described in equation (9). Foreman sequence, GOP $=4$.

Notice in Fig. 2 that encoding time of JLZA-TV is significantly smaller than all other encoders. Finally, the effect of initialization with $X^{(0)}$, see (9), is tested in Fig. 3. The comparison is made with the popular $\ell_{2}$ initialization approach, where $X^{(0)}=$ $\Phi^{\prime}\left(\Phi \Phi^{\prime}\right)^{-1} Y$. The proposed initializer reduces the iteration-count significantly.

\section{REFERENCES}

[1] D. Slepian and J. Wolf, "Noiseless coding of correlated information sources," Information Theory, IEEE Transactions on, vol. 19, no. 4, pp. 471-480, Jul 1973.

[2] A. Wyner and J. Ziv, "The rate-distortion function for source coding with side information at the decoder," Information Theory, IEEE Transactions on, vol. 22, no. 1, pp. 1-10, Jan 1976.
[3] J.-R. Ohm, "Advances in scalable video coding," Proceedings of the IEEE, vol. 93, no. 1, pp. 42-56, Jan. 2005.

[4] D. L. Donoho, "Compressed sensing," IEEE Trans. Inf. Theory, vol. 52, pp. 1289-1306, Apr. 2006.

[5] J. Romberg, "Imaging via compressive sampling," IEEE Signal Process. Mag., vol. 25, pp. 14-20, Mar. 2008.

[6] S. Cotter, B. Rao, K. Engan, and K. Kreutz-Delgado, "Sparse solutions to linear inverse problems with multiple measurement vectors," Signal Processing, IEEE Transactions on, vol. 53, no. 7, pp. 24772488, July 2005.

[7] M. Hyder and K. Mahata, "A robust algorithm for joint-sparse recovery," IEEE Signal Processing Letters, vol. 16, pp. 1091-1094, Dec 2009.

[8] W. Weerakkody, W. Fernando, and A. Adikari, "Unidirectional distributed video coding for low cost video encoding," Consumer Electronics, IEEE Transactions on, vol. 53, no. 2, pp. 788-795, May 2007.

[9] B. Girod, A. Aaron, S. Rane, and D. Rebollo-Monedero, "Distributed video coding," Proceedings of the IEEE, vol. 93, no. 1, pp. 71-83, Jan. 2005.

[10] V. Stankovic, L. Stankovic, and S. Cheng, "Compreeive video sampling," in EUSIPCO, August 2008.

[11] L.-W. Kang, A. Sinica, C.-S. Lu, and A. Sinica, "Distributed compressive video sensing," in Acoustics, Speech and Signal Processing, 2009. ICASSP 2009 Proceedings. 2009 IEEE International Conference on, April 2009.

[12] S. Lloyd, "Least squares quantization in pcm," Information Theory, IEEE Transactions on, vol. 28, no. 2, pp. 129-137, Mar 1982.

[13] E. J. Candés, J. Romberg, and T. Tao, "Stable signal recovery from incomplete and inaccurate measurements," Communications on Pure and Applied Mathematics, vol. 59, pp. 1207 - 1223, Mar. 2006.

[14] S. S. Chen, D. L. Donoho, and M. A. Saunders, "Atomic decomposition by basis pursuit," SIAM Journal on Scientific Computing, vol. 20, pp. 33-61, 1999.

[15] Y. C. Eldar and M. Mishali, "Robust recovery of signals from a structured union of subspaces," 2008. [Online]. Available: http://www.citebase.org/abstract?id=oai:arXiv.org:0807.4581

[16] H. Mohimani, M. Babaie-Zadeh, and C. Jutten, "A fast approach for overcomplete sparse decomposition based on smoothed $\ell^{0}$ norm," Signal Processing, IEEE Transactions on, vol. 57, no. 1, pp. 289301, Jan. 2009. 